\title{
Relative frequency of attribute relevance and response times in visual search*
}

\author{
GEORGE C. STONE and SHIRLEY C. PEEKE \\ Langley Porter Neuropsychiatric Institute, University of California, San Francisco, California 94143
}

\begin{abstract}
Two experiments, one using a between-Ss and one a within-Ss design, showed that response latencies to single attribute probe stimuli were longer when the target stimulus embodied two attributes (form and color) rather than a single attribute. The magnitude of this "mixed attribute effect" was influenced by the probability of attribute relevance, but the "probability effect" was mostly due to a "repetition effect," such that latencies on trials involving repetitions of the same attribute were shorter than when the relevant attribute was shifted. Implications of these results are drawn for the issues of holistic vs attributized representation of the target stimulus and serial vs parallel search of a set of attributes. Although no class of models can be ruled out on the basis of these experiments, constraints can be imposed on the versions of each that are compatible with the data presented.
\end{abstract}

This report is concerned with the ways in which humans represent simple, visually presented stimuli in short-term memory and subsequently compare the representations with information from additional stimuli to guide a judgmental response. We introduced a novel variation on familiar experimental situations by presenting a multidimensional target stimulus, followed by unidimensional probes. We examine our data in relation to three broad classes of models: (1) "extraction models" in which the initial stimuli are represented in a holistic fashion with information extracted later according to the needs of the specific task (Lockhead, 1972); (2) parallel models, in which attributized representations are interrogated simultaneously over all attributes; and (3) serial models, in which attributes are evaluated one after another. Before considering the prior state of our knowledge regarding these matters, certain methodological comments seem appropriate.

Two recent important papers by Townsend (1971, 1972) are sometimes cited as demonstrating the impossibility of distinguishing unequivocally between parallel and serial models of information processing. Townsend made no such claim. He did demonstrate that certain classes of parallel models could not be distinguished from certain other serial or hybrid models purely on the basis of the distributions of reaction times, although he gave examples of situations in which one of the formally equivalent alternatives could be rejected as psychologically implausible. A hoary tradition in psychology calls for the performing of "critical experiments": Two opposing theories are specified in considerable detail; one theory is falsified and the other is then claimed to have gained support. Townsend's papers call our attention to the fact that there are multiple alternative theories or models that could be proposed, and that individual experiments can at best

*This work was supported by Grant MH 18146 and by the California Department of Mental Hygiene. only reduce the number that need be entertained. Platt (1964), in his influential discussion of the method of inductive inference ("strong inference"), likened the process to the climbing of a tree, thereby implying a strictly hierarchical organization of possibilities in which one crucial alternative could be resolved at a time to yield final certainty with maximum efficiency. What Townsend points out is the danger that, unless all possible higher branches of the logical tree are envisioned in detail, a seemingly crucial experiment may only appear to lop off half the tree-there may remain viable alternatives hidden in the brushy top of the side supposed to be removed. The history of psychology abounds with purportedly crucial experiments that failed to resolve controversy-latent learning, continuity-noncontinuity, spatial vs response learning are a few of the topics laid aside without resolution.

In this report we attempt a slightly less ambitious goal than the final choice among three classes of models. Rather, we attempt to set limits on the versions of them that must be entertained. In the metaphor of the logical tree, our strategy is to thin it to the point where ultimate, crucial tests can be convincingly proposed.

One further methodological note needs to be struck. In our efforts to delineate the structure of the viable (not yet excluded) models, we do not assume that all persons process information in the same way, nor even that a given individual processes information in the same way at all times. Similarity between persons and between occasions must be established empirically, built up from the demonstration of such similarity from minimally pooled data. Experimentally, our task is to rule out certain types of models time after time in the hope that we can eventually assert that certain of them are "never" appropriate.

The experimental task used in this study is one of a type that has been widely studied: a target stimulus (S1) is presented to the $\mathrm{S}$, removed, and after a brief delay 
"probe stimuli" (S2) are presented. The $\mathrm{S}$ is required to make some type of judgment regarding the probe stimuli, such as "same as" or "different than" S1, whether the probe set contains S1, or which of the probe set is the same as S1. It is generally assumed in the analysis of these tasks that representations of S2 are compared in some way with stored representations of S1 as the basis for the judgment. Issues for investigation are the nature of the representation of the stimuli, the nature of the storage process, the nature of the retrieval from storage and of the comparison. The different possibilities devised to explain these matters generate the logical tree, which it is our purpose to prune.

The first branching is based upon the alternative that $\mathrm{S} 1$ is represented holistically as a unit or that it is converted to something resembling a list of its features or attributes. Lockhead (1972) has discussed this issue rather fully, concluding that humans store information as "blobs," from which they later extract or abstract the information necessary for the performance of a specific task. However, it is clear from other work that if this is the case, the $\mathbf{S}$ at least has some control over the type of blob that he stores. Such flexibility in encoding is indicated by the observation that stimuli can be matched on physical characteristics or on their nominal characteristics as well as other higher-order characteristics, such as membership in the class of vowels or consonants (Posner \& Mitchell, 1967). Aspects of the task which affect the type of encoding that is used include the duration of the interval between the two stimuli to be compared (with short intervals favoring physical codes), type of task interpolated between the stimuli [i.e., certain tasks appear to obliterate the trace required for a physical code (Posner et al, 1969)], prevention of verbal rehearsal of a name code by use of a concurrent verbal shadowing task (Kroll et al, 1972), and the S's expectation of the type of comparison to be made.

Ss' expectations have been manipulated by varying the probability that the stimulus will be in one mode as opposed to another. Tversky (1969) varied the probability that the second stimulus (S2) would be a picture or a name associated with the picture where the first stimulus (S1) could be either the corresponding picture or name or an unrelated picture or name. Her results suggest that the Ss' representations of S1 tended to favor in some way the modality of S1 (pictorial or verbal) which was most likely to appear. Frost (1972) has also concluded that the $\mathrm{S}$ can bias the type of encoding (visual or semantic) used for a given stimulus, depending on the type of task expected.

Demonstration that the accessibility of representations can be altered by the demands of the situation does not, of course, establish clearly that alternative codes are laid down. Some authors seem to favor the idea of a single code which can be transformed into a different version if necessary, while others (e.g., Frost, 1972) have argued that both codes are present but are utilized preferentially. Resolution of this question requires consideration of the manner in which multiple codes are handled, that is, whether they are accessed serially or in parallel. This branching of the logical tree has been extensively discussed in recent years (e.g., Egeth, 1966; Hawkins, 1969; Rabbitt, 1971; Saraga \& Shallice, 1973). Critical issues with regard to the parallel processing branch include the question of whether there is some limit to the amount of "processing energies" available (Atkinson, Holmgren, \& Juola, 1969; Moray, 1967; Taylor, Lindsey, \& Forbes, 1967) or whether multiple codes can be accessed without limit. ${ }^{1}$ Some studies in which the memory set consists of multiple attributes of a "single" stimulus (Hawkins, 1969; Donderi \& Case, 1970; Allport, 1971; unpublished observations from our laboratory) have found that under certain circumstances a multiattribute stimulus can be processed as rapidly and as accurately as the most slowly processed single attribute stimulus. If we knew that the S1 codes were stored as independent attributes, these results would imply that up to a point, at least, parallel processing could be accomplished without limitation, that is, that there were no "overhead costs" for maintaining and accessing two or three attributes as compared to one. However, an alternative interpretation of these studies is that the multiple attributes were encoded as proposed by Lockhead (1972) and probe stimuli compared to the "blob" without requiring further coding.

A second issue with regard to parallel processing arises only in connection with the limited capacity branch of the tree. We will phrase this question as whether or not "biasing" of parallel channels is possible. If there are limited processing energies, it is conceivable that they could be differentially assigned to multiple channels according to the demands of a particular situation. This issue has typically been raised with respect to "filtering" tasks in which one or more attributes of a multidimensional stimulus was irrelevant to the judgmental response, and thus their input to the decision process was detrimental. Garner and Felfoldy (1970) found that the requirement to filter one attribute of an "integral stimulus" resulted in longer latencies than occurred in a single attribute condition, whereas filtering of a nonintegral stimulus produced no deficit in latency. Stone (1971), using stimuli that phenomenologically would seem to be nonintegral, found that compared to single attribute stimuli, RTs were slowed by $10-30 \mathrm{msec}$ when one attribute (form) was required to be filtered and slowed by $60-120 \mathrm{msec}$ when the other attribute (color) was filtered. The results of this latter study, as well as another (Peeke \& Stone, 1973), suggest a kind of natural biasing by the nervous system with respect to color and form attributes which can be only partially overcome by the exigencies of the experiment.

Biasing is an issue also with respect to serial models, although in this case the bias would have to do with the order in which multiple attributes were processed. Some 
authors have assumed that the order of processing multiple attributes was fully under the control of the $\mathrm{S}-$ or of the experimental contingencies (Saraga \& Shallice, 1973). However, another alternative is that the order of processing is probabilistically determined and that there are limits to the amount of bias that can be induced by experimental conditions.

In the present study we used a variation of the familiar sequential judgmental task which made it possible to resolve certain points left ambiguous by other designs. We presented a condition (the "mixed attribute" condition) in which S1 had two attributes (color and form), while the probes had only a single attribute (either color or form). This condition was compared with other "single attribute" conditions, in which S1 had only the color or form attribute present. This technique allowed us to study the S's capability of biasing his storage and retrieval mechanism without the possibility that one form of code could be converted into another, as is the case with studies in which physical or verbal representations are probabilistically required. It also overcomes the difficulty presented by the filtering task in determining whether the greater response latency observed in reacting to multidimensional stimuli has to do with the storage or comparison processes or whether it may have to do only with the extraction of the relevant attribute from the probe stimuli. In the present task, since the probe stimuli are unidimensional, the latter possibility can be ruled out.

The aim of the present study was to learn more about the factors that affect the encoding of multiattribute stimuli by studying biasing factors, both "natural" biasing that occurs through comparison of RTs to color and form stimuli and biasing which is induced through varying the probability of the relevance of one attribute. Induced biasing was controlled by using three levels of probability $(.25, .50, .75)$ that the array of probe stimuli would consist of forms rather than colors. In the control condition using a single attribute $\mathrm{S} 1$, the relevant attribute was known in advance.

Two experiments were performed. In Experiment I, a different group of Ss was used with each of the three probability levels for the mixed attribute condition. All Ss received single attribute tasks based on both color and form stimuli. In Experiment II, a fully crossed within-Ss design was used with each $S$ receiving all probability levels of each attribute in each session. This design allowed a more sensitive test of the effect of probability level on the mixed attribute condition. Six sessions were presented in order to determine whether or not the effect of the biasing factors changed with practice.

\section{EXPERIMENT I}

\section{Method}

Subjects. Thirty-six Ss (19 women and 17 men) were recruited from the staff and students of the University of California, San Francisco, and California State University at San Francisco.
They ranged in age from 19 to 30 years and had normal color vision. Each $S$ participated in two sessions, each session approximately $1 \mathrm{~h}$ in length. Ss were randomly assigned to one of three groups, with 12 Ss per group.

Apparatus. The tasks were presented on the visual display programmer, a large console at which the S sat. In a horizontal row on a sloping panel were arranged the five stimulus cells (Industrial Electronic Engineers, Inline Display Cells, Series 10000 ) spaced $7.6 \mathrm{~cm}$ apart from center to center. When the $S$ was seated $40 \mathrm{~cm}$ from the panel, the five display cells subtended a visual angle of $23.5 \mathrm{deg}$ and each stimuius subtended an angle of 2.5 to $3.5 \mathrm{deg}$. Each stimulus cell (referred to as $D_{1}$ to $D_{5}$ from left to right) was covered by a transparent response panel $7.6 \mathrm{~cm} \mathrm{sq}$, referred to as $R_{1}$ to $R_{5}$. Five centimeters below $R_{3}$ was another response panel, $R_{6}$, which had no stimulus display behind it. The presentation of stimulus contingencies of the experiment was controlled by a block tape reader and associated circuitry. Each response produced an identifying record on magnetic tape together with its latency to the nearest millisecond.

Stimuli. Each color stimulus consisted of a circular patch $27 \mathrm{~mm}$ in diam of one of four colors (red, green, blue, or yellow). Each form stimulus consisted of one of four white geometric outlines (square, triangle, circle, or plus sign) approximately $20 \mathrm{~mm}$ in largest dimension centered on a dark background. To produce multiattribute stimuli, the form was superimposed on the circular color patch.

Procedure. A matching task was used where $S 1$ appeared in $D_{1}$ at the start of a trial. After examining $S 1, S$ pressed $R_{6}$ which caused $S 1$ to disappear and be replaced $180 \mathrm{msec}$ later by four probe stimuli in $D_{2}-D_{5} . S$ then pressed on the response panel corresponding to the probe stimulus that matched S1. A correct response caused the stimuli to disappear; an incorrect response had no effect on the display but was recorded. S1 for the next trial was presented $180 \mathrm{msec}$ after the correct response (i.e., as soon as the program tape could be moved).

Two conditions, distinguished by the nature of $S 1$, were run in blocks of 12 trials at a time. In the single attribute condition the S1 contained only one attribute [color $(C)$ and form $(F)$ ] and the probe stimuli consisted of the four values of the same attribute. In the mixed attribute condition (M), S1 consisted of two superimposed attributes (color and form) and the probe stimuli consisted of only one attribute, all forms $\left(\mathrm{M}_{\mathrm{f}}\right)$ or all colors $\left(M_{c}\right)$. Thus, the attribute which constituted the probe stimuli was the relevant attribute for a given trial. The $S$ did not know which attribute would be relevant on any trial in the mixed condition but was aware of the probability of relevance associated with each attribute for that block of trials.

The three groups of Ss were distinguished by the probability level for the mixed attribute condition. The probability levels were (1) $\mathrm{M}_{\mathrm{f}}=.75, \mathrm{M}_{\mathrm{c}}=.25 ;(2) \mathrm{M}_{\mathrm{f}}=.50, \mathrm{M}_{\mathrm{c}}=.50$; and (3) $\mathrm{M}_{\mathrm{f}}=$ $.25, \mathrm{M}_{\mathrm{c}}=.75$. Two sessions were presented, each session having a total of 24 blocks of 12 trials, allocated so that there were 6 blocks of $\mathrm{C}$ trials, 6 blocks of $F$ trials, and 12 blocks of $M$ trials. One mixed attribute block was interposed between each block of single attribute trials.

\section{Results and Discussion}

Median latencies were computed separately for the color-relevant trials $\left(M_{c}\right)$ and form-relevant trials $\left(M_{f}\right)$ of the mixed attribute condition and for the color trials (C) and form trials $(F)$ of the single attribute condition for each $S$ in the three probability groups. The first session served as a practice session; only the data from the second session were analyzed. Two analyses of variance for repeated measures were performed, one for color trials $\left(M_{c}\right.$ and $\left.C\right)$ and one for form trials $\left(M_{f}\right.$ and $\left.F\right)$. Each analysis of variance consisted of two variables, 
Table 1

Mean Latency (Milliseconds) of Response for Mixed Attribute Conditions for Color $\left(M_{c}\right)$ and Form $\left(M_{f}\right)$ and Single Attribute Condition for Color (C) and Form (F) and the Difference Between Them for Each Probability Level for Experiments I and II

\begin{tabular}{|c|c|c|c|c|c|c|c|c|}
\hline $\mathrm{p}^{*}$ & Group & $\mathrm{M}_{\mathrm{c}}$ & $\mathrm{C}$ & $\mathrm{M}_{\mathrm{c}}-\mathrm{C}$ & Group & $\mathrm{M}_{\mathrm{f}}$ & $\mathbf{F}$ & $M_{\mathbf{f}}-F$ \\
\hline \multicolumn{9}{|c|}{ Experiment I } \\
\hline 75 & 3 & 509.8 & 446.5 & 63.3 & 1 & 567.2 & 502.2 & 65.0 \\
\hline 50 & 2 & 573.0 & 482.2 & 90.8 & 2 & 599.7 & 537.2 & 62.5 \\
\hline 25 & 1 & 593.8 & 473.0 & 120.8 & 3 & 574.8 & 470.7 & 104.1 \\
\hline Mean & & 558.9 & 467.2 & 91.7 & & 580.6 & 503.3 & 77.3 \\
\hline \multicolumn{9}{|c|}{ Experiment II } \\
\hline 75 & & 461.2 & 422.9 & 38.3 & & 496.9 & 464.2 & 32.7 \\
\hline 50 & & 471.2 & 425.2 & 46.0 & & 511.0 & 459.8 & 51.2 \\
\hline 25 & & 492.5 & 432.3 & 60.2 & & 538.3 & 474.2 & 64.1 \\
\hline Mean & & 475.0 & 426.8 & 48.2 & & 515.4 & 466.0 & 49.4 \\
\hline
\end{tabular}

*probability of relevance

condition (mixed attribute vs single attribute) and probability level $(.75, .50, .25)$.

The main effect for conditions indicated that mixed attribute targets resulted in significantly slower RTs than single attribute targets for both color $[\mathrm{F}(1,33)=146.7$, $\mathrm{p}<.01]$ and form $[\mathrm{F}(1,33)=127.8, \mathrm{p}<.01]$. This effect will be referred to as the "mixed attribute effect" (MAE). The magnitude of the effect was of comparable size for color and form $\left(M_{c}-C=91.7 \mathrm{msec} ; M_{f}-F=\right.$ $77.3 \mathrm{msec}$ ) and varied as a function of probability level as indicated by the interaction between condition and probability group $[\mathrm{F}(2,33)=4.81, \mathrm{p}<.05$ for color; $F(2,33)=3.90, p<.05$ for form $]$.

The magnitude of the MAE for each probability level (Table 1) indicates that the effect increased with the decline of the probability level. However, the three groups differed with respect to basic speed of processing the single attribute stimuli. Since this difference in basic speed may have obscured true differences between probability levels and also since the design did not allow for direct examination of the interaction of stimulus type (color vs form) with the other variables, we decided to replicate the experiment using a within-Ss design.

\section{EXPERIMENT II}

The purpose of this experiment was to test the effect of probability level on the mixed attribute effect using a within-Ss design. It was thought that this design would provide a more sensitive test of the effects of the probability variable as well as to permit investigation (not possible in the previous experiment) of interactions of this variable with other variables such as stimulus type. Another variable of interest was the effect of practice. The Ss of the previous experiment were relatively inexperienced in processing multidimensional stimuli. We thought that extended practice might allow the Ss to perform processing operations in parallel which initially were done serially and thereby reduce or eliminate the mixed attribute effect. We also wanted to examine the effect of stimulus location on the mixed attribute effect. Previous studies from our laboratory (Peeke \& Stone, 1973), using a similar matching task, have found an interaction of stimulus type (color vs form) with stimulus location (central vs peripheral position in the display). It was thought possible that the mixed attribute effect might also interact with stimulus location. The presence or absence of such an interaction would provide information as to whether the mixed attribute effect could be accounted for by a single extra processing step that occurred once per trial or by a series of small increments (one for each comparison stimulus that was processed before the correct one was located).

\section{Method}

Subjects. The Ss were three women and one man recruited from the staff and students of the University of California, San Francisco, and California State University at San Francisco. They ranged in age from 19 to 30 years and had normal color vision. These Ss were members of a panel which had been tested weekly on the same apparatus in other tasks for a period of several months prior to this study, and hence were highly familiar with the general testing procedure and experienced in reaction time tasks. Each session was approximately $1 \mathrm{~h}$ in length, and the Ss were paid for participating.

Procedure. The apparatus, stimuli, and task were the same as that used in Experiment I. The probability levels for the relevant stimulus attribute were the same. However, in this study each $S$ received all three probability levels in each session. The probability level for a given attribute remained the same for an entire section of the experimental session. Six sessions were presented; each session was divided into three sections with each section containing one of the three probability levels. A section consisted of 24 blocks of 12 trials each with the 24 blocks allocated so that there were 6 blocks of $C$ trials, 6 blocks of $F$ trials, and 12 blocks of $M$ trials. The order in which the three probability levels were presented in a session was counterbalanced over sessions for each $\mathrm{S}$.

\section{Results}

Replication of Major Effects of Experiment I. Median latencies were computed separately for each stimulus position for the color-relevant trials $\left(\mathrm{M}_{\mathbf{c}}\right)$ and form-relevant trials $\left(M_{f}\right)$ of the mixed attribute (MA) condition and for the color trials (C) and form trials (F) of the single attribute (SA) condition for each 
Table 2

Magnitude of the Difference in Milliseconds Between Mixed Mode Condition and Single Attribute Condition for Color $\left(M_{c}-C\right)$ and Form $\left(M_{f}-F\right)$ for Each Individual at Each Stimulus Position

\begin{tabular}{|c|c|c|c|c|c|c|c|c|c|c|}
\hline \multirow[b]{3}{*}{$\mathrm{S}$} & \multicolumn{5}{|c|}{$\mathbf{M}_{\mathbf{c}}-\mathrm{C}$} & \multicolumn{5}{|c|}{$\mathrm{M}_{\mathrm{f}}-\mathrm{F}$} \\
\hline & \multicolumn{4}{|c|}{ Position } & \multirow[b]{2}{*}{ Mean } & \multicolumn{4}{|c|}{ Position } & \multirow[b]{2}{*}{ Mean } \\
\hline & 2 & 3 & 4 & 5 & & 2 & 3 & 4 & 5 & \\
\hline 3005 & 54.5 & 53.3 & 68.9 & 64.5 & 60.3 & 61.1 & 41.1 & 90.0 & 75.6 & 67.0 \\
\hline 4060 & 63.3 & 43.3 & 41.1 & 22.3 & 42.5 & 31.1 & 23.3 & 25.5 & 55.6 & 33.9 \\
\hline 24050 & 46.6 & 50.0 & 14.4 & 34.4 & 36.4 & 42.2 & 26.7 & 34.5 & 43.3 & 36.7 \\
\hline 4056 & 30.0 & 38.9 & 66.6 & 78.9 & 53.6 & 56.6 & 31.1 & 64.4 & 87.8 & 60.0 \\
\hline Mean & 48.6 & 46.4 & 47.8 & 50.0 & 48.2 & 47.8 & 30.6 & 53.6 & 65.6 & 49.4 \\
\hline
\end{tabular}

probability level and each $\mathrm{S}$. The six sessions were combined into three groups of two, representing three stages of practice. An analysis of variance for repeated measures was performed for condition (mixed attribute vs single attribute), stimulus type (color vs form), probability level of the relevant attribute $(.75, .50, .25)$, stimulus position $(2,3,4$, or 5$)$, and stages of practice.

The test of main effect for conditions indicated that, as in Experiment I, MA targets resulted in significantly slower RTs than SA targets $[F(1,3)=52.3, p<.01]$. The magnitude of the overall mixed attribute effect was very similar for color and form $\left(\mathrm{M}_{\mathrm{c}}-\mathrm{C}=48.2 \mathrm{msec} ; \mathrm{M}_{\mathrm{f}}\right.$ $-F=49.4 \mathrm{msec}$ ), and the interaction of condition and stimulus type failed to reach significance (Table 1). The MAE was found in the data of each individual $S$, although the magnitude of the effect varied among Ss; also, the magnitude of the effect was comparable for color and form for each individual (Table 2). As in Experiment I, the magnitude of the MAE was significantly affected by the probability of relevance of a given attribute $[F(2,6)=14.2, p<.01]$. The lower the probability that the attribute was relevant, the larger the MAE (Table 1). Thus, Experiment II replicates the major result of the first experiment with regard to the MAE. However, it will be shown in more detailed analyses that probability of relevance does not operate as simply as its effects on the means might suggest.

Practice Effect. A significant reduction of the MAE was found as a function of practice $[F(2,6)=6.7$, $p<.05]$. Such a practice effect probably also explains the smaller MAE found in Experiment II relative to that of Experiment I, since the Ss in Experiment II were highly experienced. Although the MAE tended to diminish with practice, it continued to be present after six sessions (see Table 3). Inspection of individual sessions indicated that the greatest effect of practice occurred prior to Session 4 with little change on subsequent sessions. The practice effect in the .25 probability condition appeared to be larger and longer lasting, but this trend did not reach significance.

Despite the significant interaction of MAE with practice, the patterns of relationships among variables were essentially the same for early (Sessions 1-3) and late (Sessions 4-6) stages of practice in many subsequent analyses. In such cases, the data were pooled over all sessions for analysis.

Position Effect. The location of the correct stimulus had a significant effect on overall latencies $[F(3,0)=$ $36.6, \mathrm{p}<.01]$; and as reported in another study using matching task (Peeke \& Stone, 1973), the magnitude of the latency differences among positions was greater for form stimuli than for color stimuli $[F(3,9)=9.2$, $\mathrm{p}<.01]$; color and form were processed at about the same speed in the center of the display, but color was processed relatively faster in the peripheral locations. Although there appears to be a trend in the data (see

Table 3

Latency (Milliseconds) for Mixed Mode Condition for Color $\left(M_{c}\right)$ and Form $\left(M_{f}\right)$ and Single Attribute Condition for Each Probability Level and Stage of Practice

\begin{tabular}{|c|c|c|c|c|c|c|c|c|c|c|}
\hline & \multicolumn{3}{|c|}{ Sessions 1-2 } & \multicolumn{3}{|c|}{$\begin{array}{l}\text { Color Trials } \\
\text { Sessions 3-4 }\end{array}$} & \multicolumn{3}{|c|}{ Sessions 5-6 } & \multirow[b]{2}{*}{ Mean } \\
\hline & $\mathrm{M}_{\mathbf{c}}$ & $\mathrm{C}$ & $\mathbf{M}_{\mathbf{c}}-\mathrm{C}$ & $\mathrm{M}_{\mathrm{c}}$ & $\mathrm{C}$ & $\mathbf{M}_{\mathbf{c}}-\mathbf{C}$ & $\mathbf{M}_{\mathbf{c}}$ & $\mathrm{C}$ & $\mathrm{M}_{\mathrm{c}}-\mathrm{C}$ & \\
\hline \multirow{5}{*}{$\begin{array}{l}75 \\
50 \\
25\end{array}$} & 486.9 & 434.4 & 52.5 & 445.6 & 416.2 & 29.4 & 451.2 & 418.1 & 33.1 & 38.3 \\
\hline & 493.1 & 441.2 & 51.9 & 457.5 & 413.8 & 43.7 & 463.1 & 420.6 & 42.5 & 46.0 \\
\hline & 511.2 & 439.4 & 71.8 & 488.1 & 421.9 & 66.2 & 478.1 & 435.6 & 42.5 & 60.2 \\
\hline & \multicolumn{3}{|c|}{ Sessions 1-2 } & \multicolumn{3}{|c|}{$\begin{array}{l}\text { Form Trials } \\
\text { Sessions 3-4 }\end{array}$} & \multicolumn{3}{|c|}{ Sessions 5.6} & \multirow[b]{2}{*}{ Mean } \\
\hline & $M_{f}$ & $\mathrm{~F}$ & $\mathbf{M}_{\mathbf{f}}-\mathrm{F}$ & $\mathbf{M}_{\mathbf{f}}$ & $\mathrm{F}$ & $M_{f}-F$ & $\mathbf{M}_{\mathbf{f}}$ & $\mathrm{F}$ & $M_{f}-F$ & \\
\hline 75 & 513.8 & 473.1 & 40.7 & 480.0 & 452.5 & 27.5 & 496.9 & 466.9 & 30.0 & 32.7 \\
\hline 50 & 533.1 & 475.6 & 57.5 & 493.8 & 445.6 & 48.2 & 506.2 & 458.1 & 48.1 & 51.2 \\
\hline 25 & 573.1 & 490.0 & 83.1 & 510.6 & 462.5 & 48.1 & 531.2 & 470.0 & 61.2 & 64.1 \\
\hline
\end{tabular}


Table 4

Mean Latency (Milliseconds) of Response for Mixed Attribute Conditions for Color $\left(M_{c}\right)$ and Form $\left(M_{f}\right)$ and Single Attribute Condition for Color (C) and Form (F) and the Difference Between Them for Each Probability Level at Each Stimulus Position

\begin{tabular}{|c|c|c|c|c|c|c|c|c|c|c|c|c|}
\hline & \multicolumn{3}{|c|}{ Stimulus Position 2} & \multicolumn{3}{|c|}{ Stimulus Position 3} & \multicolumn{3}{|c|}{ Stimulus Position 4} & \multicolumn{3}{|c|}{ Stimulus Position 5} \\
\hline & $\mathrm{M}_{\mathrm{c}}$ & $\mathrm{C}$ & $\mathrm{M}_{\mathbf{c}}-\mathrm{C}$ & $\mathbf{M}_{\mathbf{c}}$ & $\mathrm{C}$ & $\mathrm{M}_{\mathrm{c}}-\mathrm{C}$ & $\mathbf{M}_{\mathbf{c}}$ & $\mathrm{C}$ & $\mathrm{M}_{\mathrm{c}}-\mathrm{C}$ & $\mathbf{M}_{\mathbf{c}}$ & $\mathrm{C}$ & $\mathrm{M}_{\mathrm{c}}-\mathrm{C}$ \\
\hline & \multicolumn{12}{|c|}{ Color } \\
\hline $\begin{array}{l}75 \\
50 \\
25 \\
\end{array}$ & $\begin{array}{l}459.2 \\
478.3 \\
506.7\end{array}$ & $\begin{array}{l}422.5 \\
436.7 \\
439.2\end{array}$ & $\begin{array}{l}36.7 \\
41.6 \\
67.5 \\
\end{array}$ & $\begin{array}{l}404.2 \\
415.0 \\
429.2\end{array}$ & $\begin{array}{l}372.5 \\
369.2 \\
367.5\end{array}$ & $\begin{array}{l}31.7 \\
45.8 \\
61.7 \\
\end{array}$ & $\begin{array}{l}439.2 \\
449.2 \\
464.2\end{array}$ & $\begin{array}{l}398.3 \\
400.8 \\
410.0\end{array}$ & $\begin{array}{l}40.9 \\
48.4 \\
54.2\end{array}$ & $\begin{array}{l}542.5 \\
542.5 \\
570.0\end{array}$ & $\begin{array}{l}498.3 \\
494.2 \\
512.5\end{array}$ & $\begin{array}{l}44.2 \\
48.3 \\
57.5\end{array}$ \\
\hline \multirow{2}{*}{25} & $\mathbf{M}_{\mathbf{f}}$ & $\mathbf{F}$ & $M_{f}-F$ & $\mathrm{M}_{\mathrm{f}}$ & $\mathbf{F}$ & $\mathbf{M}_{\mathbf{f}}-\mathbf{F}$ & $\mathrm{M}_{\mathrm{f}}$ & $\mathrm{F}$ & $M_{f}-F$ & $\mathrm{M}_{\mathrm{f}}$ & $\mathbf{F}$ & $\mathrm{M}_{\mathbf{f}}-\mathrm{F}$ \\
\hline & \multicolumn{12}{|c|}{ Form } \\
\hline $\begin{array}{l}75 \\
50 \\
25\end{array}$ & $\begin{array}{l}514.2 \\
510.0 \\
542.5\end{array}$ & $\begin{array}{l}476.7 \\
460.0 \\
486.7\end{array}$ & $\begin{array}{l}37.5 \\
50.0 \\
55.8\end{array}$ & $\begin{array}{l}393.3 \\
400.8 \\
417.5\end{array}$ & $\begin{array}{l}372.5 \\
374.2 \\
373.3\end{array}$ & $\begin{array}{l}20.8 \\
26.6 \\
44.2\end{array}$ & $\begin{array}{l}460.0 \\
484.2 \\
512.5\end{array}$ & $\begin{array}{l}430.8 \\
431.7 \\
433.3\end{array}$ & $\begin{array}{l}29.2 \\
52.5 \\
79.2\end{array}$ & $\begin{array}{l}620.0 \\
649.2 \\
680.8\end{array}$ & $\begin{array}{l}576.7 \\
573.3 \\
603.3\end{array}$ & $\begin{array}{l}43.4 \\
75.9 \\
77.5\end{array}$ \\
\hline
\end{tabular}

Table 4) for the MAE to be smaller for the central stimulus position (3) than for peripheral positions $(2,4$, 5 ), especially for the form attribute, this interaction failed to reach significance. We kept latencies separated by stimulus position in subsequent analyses, even though we make no further mention of this variable.

Analysis of the Mixed Attribute Effect. We turn now to some analyses directed toward discovering the nature of the MAE: How does it arise, and what can it tell us about how Ss process information in these tasks?

Error Rate. One mechanism for generating an MAE would be if the $S$, on each trial, elected to store only the information about one attribute, ignoring the other. The decision as to which attribute to store could be influenced by the probability that one or the other attribute would be relevant. Such a strategy should produce high error rates on the least probable attribute. Our data on error rates (Table 5) leads us to reject this alternative since (1) the error rate is not nearly as high as it would be if only a single attribute were being stored; and (2) there was no increase in error rate for any attribute as a function of probability level.

Repetition Effects. In many situations, RTs are faster when a trial presents essentially the same conditions as those that prevailed on previous trials than they are when conditions change substantially. Although such effects have usually been observed for repetitions of a response or repetitions of an identical stimulus, a suggestion by Rabbitt and Vyas (1973) that repetition effects also occur for other phenomena (e.g., repetition of an S-R coding rule or repetition of a conceptual class) led us to consider the possibility that an "attribute-repetition effect" might exist. In our experiment, this consideration is relevant to the probability effect, since the high-probability attribute will tend to be repeated more frequently in a sequence of trials than will the lower-probability attribute.

A repetition effect would also be relevant to the MAE, however, since the single attribute condition could be viewed as having $100 \%$ probability of presenting a repetition of the relevant attribute. Slower RTs for mixed attribute tasks could thus be due entirely to an admixture of slower "shift" trials with faster "repetition" trials. We therefore tested the hypotheses that mean RTs for trials involving repetitions of an attribute were faster than those on which a shift of attribute occurred, and that the MAE and PE were due entirely to the hypothesized repetition effects. We wish to emphasize that effects due to repetition and due to probability can be conceptually distinguished. Repetition effects can exist in situations with all intertrial transition probabilities equal, while probability

Table 5

Percent Errors for Each Attribute in the Multiattribute and Single Attribute Conditions for Each Level

\begin{tabular}{|c|c|c|c|c|c|c|c|c|c|}
\hline & & \multicolumn{3}{|c|}{$\begin{array}{l}\text { Multiattribute Stimuli } \\
\text { Probability Level }\end{array}$} & & & \multicolumn{3}{|c|}{$\begin{array}{l}\text { Single Attribute Stimuli } \\
\text { Probability Level }\end{array}$} \\
\hline & & 75 & 50 & 25 & & & 75 & 50 & 25 \\
\hline \multirow[t]{2}{*}{$\mathbf{M}_{\mathbf{c}}$} & $\begin{array}{r}3005 \\
4060 \\
24050 \\
4056\end{array}$ & $\begin{array}{r}8 \\
5 \\
12 \\
14\end{array}$ & $\begin{array}{r}4 \\
5 \\
13 \\
14\end{array}$ & $\begin{array}{r}5 \\
4 \\
14 \\
12\end{array}$ & C & $\begin{array}{r}3005 \\
4060 \\
24050 \\
4056\end{array}$ & $\begin{array}{r}8 \\
5 \\
9 \\
13\end{array}$ & $\begin{array}{r}5 \\
5 \\
7 \\
11\end{array}$ & $\begin{array}{r}5 \\
3 \\
7 \\
13\end{array}$ \\
\hline & Mean & 9.8 & 9.0 & 8.7 & & Mean & 8.7 & 7.0 & 7.0 \\
\hline \multirow[t]{2}{*}{$\mathbf{M}_{\mathbf{f}}$} & $\begin{array}{r}3005 \\
4060 \\
24050 \\
4056\end{array}$ & $\begin{array}{r}9 \\
8 \\
9 \\
16\end{array}$ & $\begin{array}{r}7 \\
8 \\
15 \\
18\end{array}$ & $\begin{array}{r}9 \\
9 \\
12 \\
16\end{array}$ & $F$ & $\begin{array}{r}3005 \\
4060 \\
24050 \\
4056\end{array}$ & $\begin{array}{r}7 \\
9 \\
9 \\
15\end{array}$ & $\begin{array}{r}6 \\
8 \\
7 \\
11\end{array}$ & $\begin{array}{r}8 \\
7 \\
9 \\
13\end{array}$ \\
\hline & Mean & 10.5 & 12.0 & 11.5 & & Mean & 10.0 & 8.0 & 9.2 \\
\hline
\end{tabular}


Fig. 1. Mean RTs for individual Ss for the single attribute condition, SA, and for repetition trials, $M(R)$, and shift trials, $M(S)$, of the mixed attribute condition. RTs for color stimuli are at the top and RTs for form stimuli are at the bottom of the figure for each $\mathrm{S}$.

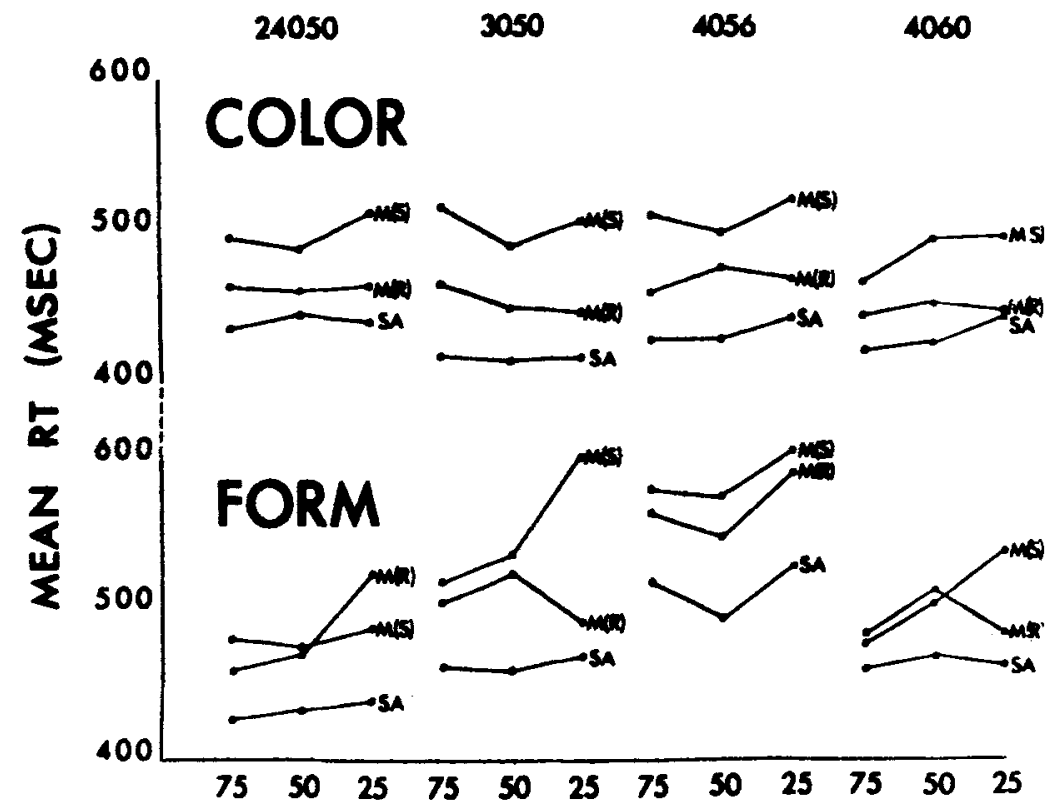

effects can exist among trials partitioned on the basis of repetition.

Mixed attribute trials were partitioned into "repetitions" (the preceding trial had the same attribute relevant) and "shifts" (the preceding trial had the other attribute relevant). ${ }^{2}$ An analysis of variance for repeated measures was performed for repetition category (repetition vs shift), stimulus type (color vs form), probability level, and stimulus location, with all sessions pooled. The main effect for the repetition category was significant $[F(1,3)=18.16, p<.05]$; the mean $R T$ for repetitions was $484 \mathrm{msec}$ and for shifts was $514 \mathrm{msec}$. Thus a modest attribute-repetition effect seemed to be present. Inspection of the data indicated large inter-S variations in patterns of response. Consequently, the next analyses were done separately for each $S$.

To test the hypothesis that the MAE might be accounted for by the repetition effect, reaction times from single attribute tasks were contrasted separately with those from "repetition" and "shift" trials. If the MAE disappeared when the repetition trials were compared with the single attribute trials (i.e., if

Table 6

Mean RTs for Repetitions and Nonrepetitions of the Same Attribute (A) for a Sequence of Three Trials

\begin{tabular}{rrrrr}
\hline \multicolumn{1}{c}{ S } & AAA & BAA & ABA & BBA \\
\hline 3005 & 469 & 461 & 491 & 511 \\
4060 & 464 & 443 & 471 & 478 \\
24050 & 462 & 454 & 477 & 484 \\
4056 & 503 & 494 & 538 & 538 \\
Mean & 475 & 463 & 494 & 503 \\
\hline
\end{tabular}

Note-AAA represents three trials with the same relevant attribute; $B A A$ represents two trials with the same relevant at tribute preceded by a trial with the other attribute relevant; $A B A$ and $B B A$ represent nonrepetitions of a given attribute $(A)$. repetition RTs were not significantly slower than single attribute RTs), then we would consider that the repetition effect could account for the MAE. Figure 1 shows the mean RTs of individual Ss for the three types of trials separately by probability of relevance for each stimulus attribute. The MAE was significant in the repetition trials of every $S$.

We then considered whether a series of repetitions of the same attribute resulted in shorter RTs than did a single repetition. The reasoning was that the single attribute condition could be considered an extended series of attribute repetitions, and if an extended series led to shorter RTs than a single repetition, this could account for the MAE. Table 6 shows that repetitions on three trials (AAA) actually produced slightly longer RTs than did single repetitions (BAA). Thus, the hypothesis that the MAE can be explained by the repetition effect was rejected.

To examine the hypothesis that the PE can be explained in terms of the repetition effect, data for individual Ss were analyzed to determine whether the PE appeared in the repetition trials, the shift trials, both, or neither. A probability effect had been obtained for both Experiments I and II when the mixed attribute trials were not distinguished according to repetitions and shifts; however, when analyses were done separately for repetitions and shifts for individual Ss, a PE was not obtained except in the shift trials of $S 4060[F(2,6)=$ $6.07, \mathrm{p}<.05]$. Although Fig. 1 displays other curves whose trend is in the direction expected if a PE were present, none of these trends was significant. A likely interpretation, therefore, is that the significant $\mathrm{PE}$ obtained for the overall MA analyses when repetition and shift trials were pooled is due to the disproportionate number of attribute repetitions (having fast RTs) in the high-probability condition and the large 
Table 7

Standard Deviations in Milliseconds of Latency Distributions of Responses at Position 3

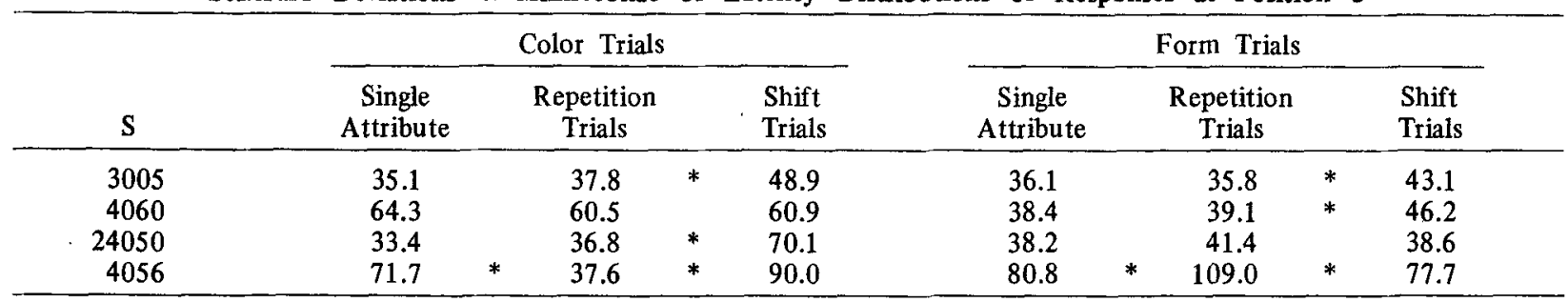

Note-Each standard deviation is pooled from six individual distributions in which stages of practice and percentage of attribute relevance were separated. Degrees of freedom associated with these pooled SDs range from 96 to 151 for the mixed attribute conditions and 246 to 306 for the single attribute conditions.

*Asterisks between columns indicate that the SDs entered in the columns differ significantly $(p<.05)$ by the $F$ test.

number of shifts (having slow RTs) in the low-probability condition. Thus, for three of four $\mathrm{Ss}$, the $\mathrm{PE}$ can be explained in terms of the RE.

Standard Deviations of Reaction Times. It is possible to make some inferences about the strategies a $S$ has used in dealing with mixed attribute tasks from comparisons of the variabilities of mixed attribute RTs with single attribute RTs. For instance, if we can demonstrate that no increase in variance is associated with a particular treatment, then the hypothesis that an additional stage of processing is required to cope with that treatment is rendered less plausible, since it is unlikely that a biological process could exist which had no variance.

There are formidable technical and theoretical difficulties concerning variability of reaction time distributions, which cannot be dealt with here. Our analyses convinced us that the usual parametric models for assessing differences between variances were often inapplicable to our situation, since they yielded a large number of significant differences in some sets of contrasts which were unsystematic in direction. We, therefore, relied primarily on a simple nonparametric approach to decide what variance estimates could be pooled. We calculated standard deviations separately for the RTs in each cell of a multidimensional array partitioned on the basis of S, stage of practice (Sessions $1-3,4-6)$, stimulus position, probability of attribute relevance, form vs color, and shift, repetition, and single attribute trials. (Data from Position 5 were dropped from this analysis because their variability was excessive.) We then contrasted the standard deviations found in parallel planes of this array. Whenever the number of positive and negative signs of the differences between the corresponding standard deviations were approximately balanced, we collapsed the array over that variable. ${ }^{3}$ In this way, we obtained variability estimates pooled over stages of practice and probability of relevance, but with each standard deviation calculated on the basis of deviation from the uncollapsed cell mean.

Even though we did not collapse variability over stimulus positions, the patterns of relationships of the variability of the latency distributions from single attribute, repetition, and shift trials were similar at all three stimulus positions. Therefore, in Table 7 , we present only the standard deviations obtained at Position 3. The major point to be noted in this table is that the standard deviations of the latency distributions for the repetition trials were with two exceptions within $4 \mathrm{msec}$ of those obtained in the single attribute trials. S 4056 showed large differences between these two conditions, which were significant at the .05 level by the $F$ test but which were opposite in direction for form and color trials. Overall, the variability in repetition trials was greater in five cases and shorter in three. Thus, it appears that there is little or no difference between the variability of responding in the single attribute condition and responding on repetition trials of the MA condition.

The situation is different with regard to the shift trials. Shift trial variabilities were larger than those of the single attribute distributions in six of eight comparisons at Position 3 and in 15 of the 16 comparisons at the other two positions. Comparison of repetition and shift trial variabilities yielded much the same pattern, in the case of color tasks. Considering all positions, 11 of 12 standard deviations of color trials were smaller for repetition tasks than for shift trials. In the form tasks the situation was also much the same, with the exception of $S 4056$, who, as can be seen in Table 7 , was significantly more variable in repetition trials than in shift trials.

To summarize, there was little or no difference in variability between the single attribute and repetition trials. Shift trial variabilities, however, were consistently larger than either single attribute or repetition trial variabilities.

\section{DISCUSSION}

In two experiments we found that when the target stimulus consists of two attributes and the probes present only one, additional time is required to process the information and select the probe stimulus that matches the target, in comparison with tasks in which both target and probe consist of only one attribute. These results can be compared with those reported by other investigators such as Sternberg (1966), who used multiple characters rather than multiattribute stimuli to manipulate memory load. Our analysis of errors ruled out the possibility that Ss stored only a single attribute. 
Studies such as those of Hawkins (1969), Donderi and Case (1970), Allport (1971) argue against the possibility that the mixed attribute effect is due to limitations in storing information from two attributes, since responses to multiattribute probes can be as fast or accurate as to single attribute probes. Rather, it would appear that the explanation should be sought in the retrieval of target information and its comparison with the information arriving from the probe stimuli.

In Experiment II we found that the mixed attribute effect diminished but did not disappear with the amount of practice given in that experiment. Both Experiments I and II demonstrated a significant relationship between the probability that an attribute was relevant in a particular block of trials and the magnitude of the mixed attribute effect. When the trials were partitioned into sets consisting of repetitions and shifts of the relevant attribute, however, it was found that most if not all of the probability effect could be attributed to a repetition effect. Almost without exception, mean latencies of response to repetitions of the same attribute were shorter than when the relevant attribute shifted between trials. Since the proportion of repetitions of an attribute was higher, when the probability of relevance was higher, a shorter mean latency was thereby generated in the high-probability conditions. There was some indication that probability of relevance affected the performance of at least one $S$ in additional ways, revealed by a significant probability effect in the analysis where repetitions and shifts were considered separately.

The mixed attribute effect cannot be attributed simply to the operation of the repetition effect, since the MAE was demonstrated with a high degree of significance in the data of each $\mathrm{S}$, even when only repetition trials were considered.

What conclusions can be drawn about the storage and retrieval of representations of multiple attributes from the results of this experiment? Consider first the implication for models involving extraction of information from a holistic code. All extraction models imply an increase in the mean reaction time in the mixed attribute situation because of the addition of an extraction stage to the processing time. This increase was found for all of our Ss. However, extraction models also imply an increase in the variance of the latency distributions in the mixed attribute situation, unless one is willing to consider that there exists a time-consuming biological process which has no variability. Since one of our Ss (S 3005) showed consistently smaller variances in the mixed attribute situation than in the single attribute situation (when all response positions are considered), we can rule out an extraction model in accounting for her data. Furthermore, for those Ss for whom we cannot rule out an extraction process, we can make the assertion that if one exists, it has the property of being affected by the recency or frequency of presentations of the attribute being extracted. This assertion is based on our demonstration of a repetition effect and possibly a probability effect over and above the repetition effect. However, the fact that a viable version of an extraction model cannot be ruled out for some Ss does not preclude viability for other models.

Consider next a serial search model. We may ask the question of whether the two attributes are searched in some specifiable order or whether there is a random, probabilitistic order of search. The latter alternative is implausible to the same degree as were the extraction models on the grounds that if the first attribute to be evaluated is chosen probabilistically, then on some trials it will be the irrelevant attribute and the second attribute must be searched (yielding a longer latency). This mixture of short and long latencies in the reaction time distribution would increase the variance in the mixed attribute situations over that of the single attribute situations, a result which was not obtained.

In the branch of models involving fixed-order serial search, there are many possible rules for determining which attribute to evaluate first. Uniform priority given to one attribute, for example color, would lead one to expect little or no MAE for the preferred attribute. This possibility appears to be excluded on the basis of our finding that the mixed attribute effect was comparable in magnitude for form and color. While one could explain the occurrence of a mixed attribute effect in the preferred attribute on the basis of some kind of overhead cost, or divided attention effect resulting from dealing with two attributes, such an effect ought certainly to be operative on the second attribute as well as the first, and in the case of the second, there would be the additional time required to complete the first evaluation. Actually, close inspection of Table 5 indicates that, at Position 3, the mixed attribute effect for form was consistently slightly less than that for color, while at the other positions it tended to be longer. Only an unappealingly complex preferred-attribute model could accommodate these results.

Another fixed-order model that readily suggests itself is one in which the $S$ always processes first the attribute which is most likely to be relevant in that block of trials. This rule will not, of course, determine what to do when the probability of relevance is $50 \%$ for both attributes, so some secondary rule must be applied in that situation. However, overlooking this difficulty, this model comes down to a double preferred-attribute model. Such a model has all of the deficiencies already noted for the single preferred-attribute model. Furthermore, it implies a much stronger probability effect than we obtained.

A third fixed-order model is based on the decision rule to evaluate first the attribute that was relevant on the previous trial. This model would generate the prediction that the mean and variance of latencies on the repetition trials would be less than those of the shift trials, which they were in our data. The presence of the mixed attribute effect in the repeated attribute trials requires the addition of an overhead cost assumption to this model. This model fails to provide any basis for a 
further probability effect such as was seen in the data of one of the Ss. However, it appears to be more plausible than either of the other fixed-order models considered here.

No unlimited parallel search model is viable because of the existence of the mixed attribute effect. To be viable, a limited capacity parallel model must make some provision for enhancement of the evaluation of a particular attribute by a recent success with it. Since parallel models, by definition, assume that both attributes are evaluated on every trial, one cannot invoke a repetition effect produced simply by recency of use. Therefore, there must exist some unavoidable bias between the parallel channels, that is, some difference in the priority assigned to their information.

In summarizing, we make the following assertions about the information processing exhibited in our experiments: (1) Some Ss did not employ an extraction process subsequent to the presentation of S2. (2) Some Ss did not employ a probabilistically ordered serial retrieval mechanism. (3) No S (in Experiment II) showed a uniform preference for processing one attribute before the other. (4) No S exhibited an unlimited, parallel retrieval process. (5) No $S$ exhibited an unbiased, limited parallel retrieval process.

Conclusions 1 and 4 taken together with the demonstrations (cited earlier) that multidimensional stimuli can be processed as quickly as unidimensional stimuli suggest that blob processing is an optional strategy available to $\mathrm{Ss}$ under some conditions. Conclusions 2 and 3 suggest that if serial retrieval is employed, it is under voluntary control, but that Ss vary their strategies for ordering in complex ways. But the results in filtering tasks indicate that there are limits to the possibility of determining order. Conclusions 4 and 5 imply that if people use a parallel retrieval process, it is both limited and biased.

We must emphasize once more that the conclusions we draw from these experiments regarding how the information was processed are not necessarily applicable to other tasks, other conditions, or even to other Ss. Saraga and Shallice (1973) argue that it is unnecessarily pessimistic to assume that "the normal mode of operation of any component process will not be found to be either parallel or serial [p. 261]." We believe that the burden of proof lies on the optimist.

\section{REFERENCES}

Allport, D. A. Parallel encoding within and between elementary stimulus dimensions. Perception \& Psychophysics, 1971, 10, 104-108.

Atkinson, R. C., Holmgren, J. E., \& Juola, J. F. Processing time as influenced by the number of elements in a visual display. Perception \& Psychophysics, 1969, 6, 321-326.

Donderi, D., \& Case, B. Parallel visual processing: Constant same-different decision latency with two to fourteen shapes. Perception \& Psychophysics, 1970, 8, 373-375.

Egeth, H. E. Parallel vs serial processes in multidimensional stimulus discrimination. Perception \& Psychophysics, 1966, 1, 245-252.

Frost, N. Encoding and retrieval in visual memory tasks. Journal of Experimental Psychology, 1972, 95, 317-326.

Garner, W. R., \& Felfoldy, G. L. Integrality of stimulus dimensions in various types of information processing.
Cognitive Psychology, 1970, 1, 225-241.

Hawkins, H. L. Parallel processing in complex visual discrim ination. Perception \& Psychophysics, 1969, 5, 56-64.

Kroll, N. E. A Parkinson, S, R \& Parks, T. E. Sensory and active storage of compound visual and auditory stimuli. Journal of Experim ental Psychology, 1972, 95, 32-38.

Lockhead, G. R. Processing dimensional stimuli: A note. Psychological Review, 1972, 79, 410-419.

Moray, $N$. Where is capacity limited? A survey and model. Acta Psychologica, 1967, 27, 84-92.

Peeke, S. C., \& Stone, G. C. Focal and nonfocal processing of color and form. Perception \& Psychophysics, 1973, 14, 71-80.

Platt, J. R. Strong inference. Science, 1964, 146, 347-352.

Posner, M. I., Boies, S. J., Eichelman, W. H., \& Taylor, R. L. Retention of visual and name codes of single letters. Journal of Experim ental Psychology, 1969, 79(1, Pt. 2).

Posner, M. I., \& Mitchell, R. F. Chronometric analysis of classification. Psychological Review, 1967, 74, 392-409.

Rabbitt, P. M. A. Times for analyzing stimuli and selecting resp onses. British Medical Bulletin, 1971, 27, 259-265.

Rabbitt, P. M. A., \& Vyas, S. What is repeated in the "repetition effect." In Attention and performance IV. New York: Academic Press, 1973.

Saraga, E., \& Shallice, $R$. Parallel processing of the attributes of single stimuli. Perception \& Psychophysics, 1973, 13, 261-270.

Sternberg, S. High-speed scanning in human memory. Science, $1966,153,652-654$.

Stone, G. C. Response latencies in visual search involving redundant or irrelevant information. Perception \& Psy chophysics, 1971, 9, 9-14.

Taylor, M. M., Lindsay, P. H., \& Forbes, S. M. Quantification of shared capacity processing in auditory and visual discrimination. Acta Psychologica, 1967, 27, 223-229.

Townsend, J. T. A note on the identifiability of parallel and serial processes. Perception \& Psychophysics, 1971, 10, 161-163.

Townsend, J. T. Some results concerning the identifiability of parallel and serial processes. British Journal of Mathematical \& Statistical Psychology, 1972, 25, 168-199.

Townsend, J. T. A note on the identifiability of parallel and serial processes. British Journal of Mathematical \& Statistical Psychology, in press.

Tversky, B. Pictorial and verbal encoding in a short-term mem ory task. Perception \& Psychophysics, 1969, 6, 225-233.

\section{NOTES}

1. Since the completion of this manuscript, Kahneman's excellent review and critique of limited capacity models has become available. Several of the ideas we tentatively propose would have benefited from reexamination in light of his theory. 2. We attempted to separate further those trials which involved repetitions of an identical stimulus and an identical response. The number of trials in both of these categories was very small, and no significant effects were discovered. There was a fairly consistent tendency for repetition of the identical stimulus to yield slightly faster RTs; repeated responses were stimulus to yield slightly faster RTs; repeated responses were systematically slower for the other two. A separate analysis was performed on "repetitions" with trials involving repeated stimuli removed; differences from analyses reported here were negligible.

3. For example, we first contrasted variabilities from the early and late sessions. This contrast was based upon 216 pairs of standard deviations, of which 115 were larger in the early sessions, 96 in the later sessions, and 5 were indeterminant. Since there appeared to be no pronounced trends within the subdivisions of the array (suggestive of interactions), we felt justified in pooling variance estimates over the stages of practice. We then considered whether it was similarly possible to pool over stimulus positions, over percentages of relevance, and over the form vs color distinction. Of these, only the probability of attribute relevance yielded balanced signs. In the contrast between the $75 \%$ and $25 \%$ conditions, 32 SDs were larger in $75 \%$ conditions and 36 were larger in $25 \%$ conditions. Similarly, in contrasting the $75 \%$ and $50 \%$ conditions, 37 contrasts showed larger standard deviations in the $75 \%$ conditions and 34 in the $50 \%$ conditions. We therefore collapsed our variability array over the percentage relevance variable. No further collapsing was possible: variabilities were smallest at Position 3, larger at Position 4, and langest at Position 2. Variabilities were consistently larger in responding to form trials than in responding to color trials at Positions 2 and 4 , although not at Position 3. The differences between the single attribute and multiple attribute trials were the focus of our interests.

(Received for publication October 22, 1973; accepted November 28,1973 .) 